\title{
Gravidez após cirurgia bariátrica: uma revisão bibliográfica
}

\author{
Pregnancy after bariatric surgery: a literature review
}

\author{
Embarazo tras cirugía bariátrica: revisión de la literatura
}

Thaiz Geovana Bezerra ${ }^{1 *}$, Beatriz Beniz Alves Caldeira², Thays Araújo Rocha Bitencourt ${ }^{3}$, Eloísa Lee Lelis $^{4}$, Larissa Hermann de Siqueira Damas de Andrade ${ }^{5}$, Ludmila Ferrante Silveira Maia ${ }^{6}$, Luna Hussein Colombelli ${ }^{7}$, Maria Fernanda Rebello Vieira ${ }^{6}$, Maria Shayany de Sousa Soares ${ }^{8}$ Wagner Pablo Corrêa ${ }^{9}$.

\section{RESUMO}

Objetivo: Descrever as complicações relacionadas à gestação após a cirurgia bariátrica e sua relação com as repercussões perinatais. Revisão bibliográfica: Observou-se que a maioria das cirurgias bariátricas em mulheres ocorre em idade reprodutiva. Atualmente, não há consenso sobre qual o momento ideal para engravidar após uma cirurgia bariátrica, mas aconselha-se esperar cerca de 2 anos, para que haja uma gravidez segura. Pacientes submetidos ao bypass gástrico, possuem risco elevado de desenvolver deficiências vitamínicas (A, D, B12, B9 e selênio). Pode haver, ainda, complicações como obstrução intestinal, hemorragia digestiva, deiscências de anastomoses e hérnias. Entretanto, a intervenção cirúrgica prévia à gestação é benéfica e está associada a melhora fertilidade, além de uma menor incidência de diabetes gestacional, doença hipertensiva da gravidez, prematuridade, necessidade de parto cesáreo, anormalidades de crescimento fetal e mortalidade perinatal Considerações finais: Reforça-se a importância da discussão acerca dos riscos e benefícios da gestação em mulheres após a cirurgia bariátrica, bem como planejamento de diretrizes para cuidados desde a pré concepção até o puerpério, tanto das mulheres quando dos neonatos.

Palavras-chave: Cirurgia bariátrica, Gravidez, Complicações.

\begin{abstract}
Objective: Describe pregnancy-related complications after bariatric surgery and their correlation with adverse perinonic repercussions. Bibliographic review: It was observed that most bariatric surgeries in women occur at reproductive age. Currently, there is no medical consensus on the ideal time to become pregnant after a bariatric surgery but, it is advisable to wait about 2 years, because it is a phase of high catabolism. Patients undergoing gastric bypass have a high risk of developing vitamin deficiencies ( $A, D, B 12, B 9$ and selenium). Furthermore, there may be complications such as intestinal obstruction, gastrointestinal bleeding, dehiscence of anastomoses, herniations or band eons. However, surgical intervention is beneficial, being associated with fertility in women, who were previously obese, and who had difficulty getting pregnant, in addition to a lower incidence of gestational diabetes. According to a meta-analysis of 33 studies, it was concluded that women who had bariatric surgery of childbearing age had pregnancy with high perinatal mortality, prematurity, congenital and mental anomalies, which may be related to nutritional malabsorption and hypovitaminosis.
\end{abstract}

\footnotetext{
${ }^{1}$ Universidade do Oeste Paulista, Jaú - SP. *E-mail: thaizgeovanabezerra@gmail.com

${ }^{2}$ Centro Universitário de João Pessoa (UNIPÊ), João Pessoa - PB.

3 Universidade Católica de Pernambuco (UNICAP), Recife - PE.

${ }^{4}$ Faculdades Integradas do Norte de Minas (FUNORTE), Montes Claros - MG.

${ }^{5}$ Centro Universitário Tocantinense Presidente Antônio Carlos (UNITPAC), Araguaína - TO.

${ }^{6}$ Faculdade Atenas Passos (Uniatenas), Passos - MG.

7 Universidade Católica de Pelotas (UCPel), Pelotas - RS.

${ }^{8}$ Faculdade Metropolitana São Carlos (Famesc), Bom Jesus do Itabapoana - RJ.

9 União Educacional do Vale do Aço (Univaço), Ipatinga - MG.
} 
Final considerations: Thus, it can be seen that the discussion about complications and benefits is appropriate, for there to be, a planning of the care of women and babies, reducing the risk and increasing the quality of life.

Keywords: Bariatric surgery, Pregnancy, Complications.

\section{RESUMEN}

Objetivo: Describir las complicaciones relacionadas con el embarazo tras la cirugía bariátrica y su correlación con las repercusiones perinatales adversas. Revisión bibliográfica: Se observó que la mayoría de cirugías bariátricas en mujeres ocurren en edad reproductiva. Actualmente, no existe un consenso médico sobre el momento ideal para quedar embarazada después de una cirugía bariátrica, pero es recomendable esperar unos 2 años, ya que es una fase de catabolismo. Los pacientes sometidos a bypass gástrico tienen un alto riesgo de desarrollar deficiencias de vitaminas ( $A, D, B 12$, B9 y selenio). Además, puede haber obstrucción intestinal, hemorragia digestiva, dehiscencia de anastomosis, hernia o erosión de la banda. Sin embargo, la intervención quirúrgica es beneficiosa, ya que se asocia con la fertilidad en mujeres que anteriormente eran obesas y tenían dificultades para quedar embarazadas, además de una menor incidencia de diabetes gestacional. Según un metaanálisis de 33 estudios, se concluyó que las mujeres que tenían cirugía bariátrica en edad fértil tenían un embarazo con alta mortalidad perinatal, prematuridad, anomalías congénitas y mentales, que pueden estar relacionadas con malabsorción nutricional e hipovitaminosis. Consideraciones finales: De esta manera, se ve que la discusión sobre complicaciones y beneficios es adecuada, para que exista una planificación de la atención a mujeres y bebés, reduciendo el riesgo y aumentando la calidad de vida.

Palabras clave: Cirugía bariátrica, El embarazo, Complicaciones.

\section{INTRODUÇÃO}

A cirurgia bariátrica é a intervenção cirúrgica indicada para solucionar casos de obesidade que não obtiveram sucesso com o tratamento clínico, que inclui, por exemplo, mudanças no estilo de vida e na alimentação. A população obesa vem crescendo exponencialmente e, com benefícios a curto e longo prazo, a cirurgia bariátrica é uma forma eficiente de se alcançar a perda de peso e a redução de comorbidades, tais como a Diabetes Mellitus tipo II, hipertensão arterial sistêmica e síndrome metabólica, além de exercer efeitos favoráveis no funcionamento de vários sistemas do organismo (FALCONE V, et al., 2018).

À medida que um número notável de mulheres em idade reprodutiva vem sendo submetidas a essa cirurgia, cresce também a ocorrência de gestações após o procedimento, o que torna necessário desenvolver um acompanhamento rigoroso gineco-obstétrico (COELHO A, et al., 2020). A obesidade repercute negativamente na gravidez, acarretando um maior tempo até a concepção, bem como complicações para as saúdes materna e fetal. Sendo assim, a perda de peso mediada pela cirurgia bariátrica pode promover desfechos desejáveis sobre a saúde reprodutiva e metabólica dessas mulheres (CARREAU AM, et al., 2017).

As possíveis complicações decorrentes das mudanças no trato digestivo após a cirurgia bariátrica, por outro lado, também devem ser consideradas (STOPP T, et al., 2018). Nesse sentido, são descritos resultados adversos que envolvem, sobretudo, as deficiências de micronutrientes como a vitamina $B_{12}$, vitamina $D$, cálcio, folato e ferro; macronutrientes, principalmente proteínas e lipídios; síndrome de dumping; complicações cirúrgicas como hérnias intra-abdominais; partos prematuros e crianças pequenas para idade gestacional (PIG) ou grandes para a idade gestacional (GIG), além de demais complicações (FALCONE V, et al., 2018; COELHO A, et al., 2020).

Mesmo diante dos riscos já documentados, a literatura ainda carece de informações bem definidas de forma a embasar o manejo apropriado das mulheres após a cirurgia bariátrica, desde o planejamento concepcional até a gestação. Assim, o aconselhamento clínico-cirúrgico, torna-se um desafio crescente para cirurgiões e obstetras (CARREAU AM, et al., 2017). 
O presente artigo, portanto, tem como objetivo reunir evidências científicas atuais a respeito dos potenciais riscos durante a gravidez após cirurgia bariátrica em mulheres férteis e a sua associação com resultados perinatais adversos.

\section{REVISÃO BIBLIOGRÁFICA}

\section{Técnicas de cirurgias bariátricas}

A maioria das mulheres submetidas ao processo de cirurgia bariátrica estão em idade reprodutiva, havendo a possibilidade de gestação após a realização do procedimento. Com isso, se torna necessários a abordagem dos riscos, complicações e benefícios de cada intervenção em mulheres que têm potencial de desenvolver uma gestação, principalmente pelo fato de existirem avanços nas técnicas cirúrgicas como, por exemplo, o uso da laparoscopia e da cirurgia robótica, que representa uma alternativa para esse tipo de procedimento. As opções atuais para a realização da cirurgia bariátrica compreendem três intervenções que são descritas como: restritivas, disabsortivas e mistas (ABENHAIM HA, et al., 2018; ADAM S, et al., 2017).

As cirurgias restritivas, dividem-se nos subgrupos bypass jejunoileal tradicionais, banda gástrica ajustável e a gastroplastia/gastrectomia vertical. A técnica do bypass jejunoileal tradicional busca promover o desvio intestinal do jejuno até o íleo com a colocação de um anel restritivo. Já o LAGB é um procedimento geralmente realizado por videolaparoscopia, em que se coloca um dispositivo tipo anel gástrico ajustável formando uma bolsa na parte superior do estômago, havendo assim uma diminuição artificial do tamanho do estômago. Essa banda será conectada por uma via subcutânea podendo ser ajustada posteriormente. A desvantagem dessa técnica está relacionada ao deslizamento do material que pode causar oclusão completa ou erosões (MONSON M e JACKSON M, 2016).

A gastrectomia vertical, por sua vez, é o procedimento mais atual e o segundo mais realizado, representando $20 \%$ do total dos procedimentos. Esses procedimentos restritivos são mais simples de serem realizados resultando na saciedade precoce, prolongamento do tempo de esvaziamento gástrico e consequente redução na ingestão alimentar. $O$ fato de ser uma modalidade restritiva e não afetar de forma tão significativa a absorção dos nutrientes representa uma boa alternativa para mulheres em idade fértil pela necessidade de nutrição adequada durante o período da gestação (ADAM S, et al., 2017; COLQUITT J, et al., 2014).

As cirurgias com mecanismo disabsortivo, possuem duas técnicas realizadas, o desvio jejunoileal e a derivação biliopancreática. Esses procedimentos são baseados em modelos que encurtam a superfície total de absorção através da redução da parte do intestino delgado, e como consequência promove menor absorção (ROBSON S, et al., 2015).

Nas cirurgias com mecanismo misto, que associa o componente restritivo e o disabsortivo, temos como exemplo o bypass gástrico em $Y$ de Roux ou desvio biliopancreático. Esta técnica é a mais realizada, perfazendo $54 \%$ do total dos procedimentos e tem como consequência a síndrome do intestino curto que leva à absorção lentificada e reduzida dos nutrientes, bem como alterações hormonais de grelina, responsável por modular a fome, de peptídeo YY e Glucagon-Like Peptide-1(GLP-1), os quais suprimem o apetite. No entanto, esse modelo tem como possíveis complicações a ocorrência de fístula e úlcera gástrica, dentre outros (ADAM S, et al., 2017; MONSON M e JACKSON M, 2016; ROBSON S, et al., 2015; COLQUITT J, et al., 2014).

Quando se trata de pacientes que têm o desejo de ter filhos, elas são orientadas a aguardar dois anos após a realização da cirurgia bariátrica para engravidar. No entanto, faltam evidências quanto a essa orientação (ROBSON S, et al., 2015). No que diz respeito às complicações gestacionais relacionadas a mulheres que realizaram a cirurgia bariátrica, existem estudos demonstrando prematuridade, necessidade de parto cesáreo e recém-nascido PIG (STUART A e KÜLLEN K, 2017).

A realização da cirurgia bariátrica está atrelada a menor incidência de diabetes mellitus gestacional e de peso aumentado para idade gestacional (STUART A e KÜLLEN K, 2017). Desse modo, por existirem resultados conflitantes a respeito dos neonatos, das gestantes, e somado ao crescente número de cirurgias bariátricas realizadas, é válida a discussão dos riscos e benefícios, bem como planejamento de diretrizes para cuidados desde a pré-concepção até o puerpério, tanto das mulheres quando dos recém-nascidos (ABENHAIM HA, et al., 2018; ADAM S, et al., 2017). 
Entre as técnicas cirúrgicas analisadas, os procedimentos restritivos se mostram relacionados com as deficiências de micronutrientes. No entanto, a suplementação no primeiro trimestre de gestação é significativa na saúde materna e fetal independente da técnica utilizada para a realização da cirurgia bariátrica (HAZART J, et al., 2017).

\section{Mudança na Fertilidade}

A procura e indicação pela cirurgia bariátrica é crescente em mulheres jovens com obesidade grave em idade reprodutiva (COELHO A, et al., 2020). De acordo com alguns casos de mulheres que apresentavam alguma dificuldade na tentativa de engravidar antes da cirurgia bariátrica obtiveram êxito após o procedimento, insinuando a interferência benéfica sobre a fertilidade feminina (HERNANDEZ-PINZON J, et al., 2008; MANCINI MC, 2016). As pacientes que não possuem o interesse em engravidar, deve ter atenção aos riscos de ter uma gestação indesejada após os procedimentos, visto que, a perda de peso geralmente recompõe aceleradamente os ciclos ovulatórios e pode diminuir a eficácia e absorção dos anticoncepcionais de via oral, o que diminui a eficácia dos fármacos de anticoncepção (COELHO A, et al., 2020).

A anticoncepção é recomendada por um período de 24 meses após a intervenção cirúrgica, em que se faz necessária durante esse tempo devido a possíveis complicações na ocorrência de uma gestação em mulheres que se submeteram a cirurgia bariátrica. Contudo, mesmo diante dessas condições há pouca adesão por mulheres em idade fértil. Desse modo, tem-se a importância do aconselhamento anticoncepcional e 0 esclarecimento dos riscos gestacionais em mulheres em idade fértil que passaram por cirurgia bariátrica de forma a favorecer a adesão da anticoncepção nesse período (MENKE MN, et al., 2017; NARAYANAN RP e SYED AA, 2016).

Sabe-se também que mulheres com obesidade possuem, muitas vezes, síndrome dos ovários policísticos (SOP), que pode ser responsável pelas dificuldades de alcançar uma gravidez. Nesses casos, a realização da cirurgia bariátrica em mulheres em idade fértil pode contribuir de maneira significativa para a gestação, pois após a intervenção cirúrgica os efeitos são positivos na SOP (CARREAU AM, et al., 2017). Essa melhora ocorre devido à perda de peso, que pode variar de 50 a 75\% a depender do estudo analisado, e que possibilita uma mudança hormonal favorável à regulação de hormônios que antes eram desajustados. (ADAM S, et al., 2017).

\section{Consequências Gestacionais}

Durante a gestação, a obesidade representa um agravante para possíveis riscos maternos e fetais. Frente a isso, reforça-se a importância da intervenção médica antes da concepção, minimizando as chances de parto prematuro, diabetes gestacional e hipertensão induzida pelas condições de obesidade durante o período gestacional (HERNANDEZ-PINZON J, et al., 2008). A cirurgia bariátrica pode proporcionar uma mudança nesse contexto, e mesmo nos casos onde é realizada, as mulheres podem permanecer acima do peso ideal, evidenciando a indispensabilidade do acompanhamento obstétrico pré-natal (MANCINI MC, 2016).

A identificação das possíveis complicações durante o período gestacional em mulheres que já realizaram a cirurgia bariátrica se faz necessário durante o pré-natal. Os sintomas habituais da gravidez podem estar relacionados a complicações graves para a paciente e para a gestação, entre eles: obstrução intestinal, deiscência de anastomoses, existência de hérnias ou erosão de banda. Estes, por sua vez, necessitam de uma avaliação minuciosa e maior atenção do profissional que assiste tais pacientes, pois as complicações têm o risco de mortalidade materna se não identificadas de forma precoce (ADAMS T, et al., 2015). A importância do diagnóstico se dá uma vez que, na presença de tais complicações, a mortalidade materna pode chegar a $20 \%$ (COELHO A, et al., 2020).

O surgimento de hérnias é uma consequência após o bypass gástrico que pode chegar a $5 \%$, que tem risco aumentado em mulheres grávidas após a cirurgia bariátrica que está relacionado com o aumento da pressão intra-abdominal exercida pelo útero no intestino. Com isso, ocorre a possibilidade de abdome agudo caso a herniação seja interna, o que leva a necessidade de um diagnóstico oportuno para que se previne complicações maternas como a necrose intestinal e intercorrências fetais (STOOP T, et al., 2018). 
Além disso, pacientes que engravidaram logo após o bypass gástrico manifestam grande risco em desenvolver deficiências vitamínicas ( $A, D, B 12, B 9$, selênio), além de outras complicações como obstruções intestinais e hemorragia digestiva (MANCINI MC, 2016; COELHO A, et al., 2020). As síndromes de dumping podem ser uma consequência da bariátrica e que precisam ser distinguidas dos sintomas comuns de uma gravidez normal. O dumping precoce é decorrente do rápido trânsito alimentar gastrointestinal, enquanto o dumping tardio é caracterizado por uma resposta hiperinsulinêmica à rápida absorção de carboidratos simples. Dessa forma, o diagnóstico é necessário para que se estabeleça o tratamento através de medidas dietéticas e farmacológicas para o tratamento da síndrome de dumping (NARAYANAN RP e SYED AA, 2016).

São descritos, ainda, diversos malefícios da obesidade em gestantes. Dentre eles, destacam-se a doença hipertensiva da gravidez, diabetes gestacional, prematuridade, necessidade de parto cesáreo, anormalidades de crescimento fetal e mortalidade perinatal quando comparado a gestantes não obesas. Uma coorte retrospectiva realizada nos Estados Unidos, analisou dados de partos hospitalares e dividiu as puérperas em 3 grupos: pacientes que realizaram bariátrica anteriormente à gestação, pacientes obesas de grau III e aquelas que não se enquadram em nenhuma dessas opções. Quando comparados os dois primeiros grupos, observou-se menos complicações gestacionais, menor número de parto cesáreo ou instrumental, menos hemorragia, além do menor tempo de internação naquelas que tinham realizado cirurgia bariátrica previamente (ABENHAIM HA, et al., 2018).

Além da redução da incidência de diabetes gestacional em mulheres que engravidam após cirurgia bariátrica, há também a redução de distúrbios hipertensivos e macrossomia, quando comparado com mulheres que apresentam obesidade durante a gestação (UPALA S e ANGUANKEO A, 2016). Reforça-se ainda que os cuidados no período de puerpério ainda se fazem necessários mediante ajustes terapêuticos e a necessidade da administração de polivitamínicos por via oral ou parenteral, visto que, o processo de absorção de nutrientes no trato digestivo pode estar prejudicado na maioria dos casos (COELHO A, et al., 2020).

\section{Consequências fetais}

De acordo com os resultados maternos em relação ao seu estado nutricional e estrutural, os efeitos fetais negativos e positivos são significativos em recém-nascidos filhos de mulheres que engravidam após cirurgia bariátrica. O diabetes gestacional e hipertensão são exemplos de fatores que se encontram relacionados a piores consequências em pacientes com obesidade, que reflete no desenvolvimento e no curso gestacional. Contudo, podem estar ausentes ou diminuídos em pacientes que já passaram por procedimento cirúrgico bariátrico, o que implica em consequência positivas para o feto, como a redução de peso médio dos recémnascidos, verificando-se uma maior incidência de recém-nascidos PIG, o que gera consequências durante e após ao nascimento (RAM PN e SYED AA, 2016).

Contudo, mulheres grávidas após o procedimento de cirurgia bariátrica têm a chance de desenvolver patologias que podem interferir na formação e desenvolvimento fetal, visto que, a capacidade de gerar macrossomia podem afetar o período de gestação acarretando na sua interrupção. A má formação fetal é decorrente de alterações no rearranjo cromossômico, que tem mais possibilidade em mães que já passaram pelo procedimento de cirurgia bariátrica devido a carência nutricional relacionada a não suplementação de micronutrientes e macronutrientes (HAZART J, et al., 2017).

Akhter Z, et al. (2019) apresentaram uma meta-análise, que foi elaborada a partir de 33 estudos, com uma visão atualizada sobre a gestação após a cirurgia bariátrica. Os autores concluíram que mulheres que fizeram essa cirurgia em idade fértil apresentaram gestação com maior frequência de mortalidade perinatal, recémnascidos pré-termo, anomalias congênitas e mentais, fetos PIG e maiores chances de admissão em Unidade de Terapia Intensiva Neonatal. Esses efeitos adversos podem estar relacionados, entre outras causas, à desnutrição que é frequentemente associada a procedimentos que geram má absorção para a gestante, principalmente, para aquelas que foram submetidas aos procedimentos como o bypass jejunocólico e 0 bypass jejunoileal. (AKHTER Z, et al., 2019). A cirurgia bariátrica é responsável por reduzir o risco de bebês GIG se comparado com gestantes que estão acima do peso (RÓŻAŃSKA-WALĘDZIAK A, et al., 2020). 
A influência da cirurgia bariátrica na captação de nutrientes resulta em uma menor absorção de micronutrientes e macronutrientes importantes para a manutenção da homeostase materna e desenvolvimento do feto até o seu nascimento. A deficiência de vitamina B12, decorrente da falta de sua captação durante a dieta materna, também foi observada em mulheres submetidas a cirurgia bariátrica (STOPP T, et al., 2018). Embora os dados disponíveis ainda sejam conflitantes, a deficiência de vitamina B12 parece estar relacionada a um maior risco de parto prematuro, aborto recorrente, baixo peso ao nascer, retardo de crescimento intrauterino, defeitos do fechamento do tubo neural e desenvolvimento cognitivo prejudicado. Assim os obstetras devem analisar os níveis de vitamina B12 de após a realização da cirurgia bariátrica a cada trimestre e tratar a sua deficiência através de uma suplementação adequada de acordo com as suas necessidades (FALCONE V, et al., 2018).

A deficiência de ácido fólico merece atenção especial devido a sua relação com defeitos neurológicos severos e frequentes em recém nascidos. A carência de ácido fólico afeta o desenvolvimento do tubo neural, o que torna importante a suplementação antes e durante o período gestacional de pacientes que já se submeteram ao procedimento de cirurgia bariátrica. A falta de vitamina A no organismo materno também é uma contribuinte para as causas de alterações no sistema nervoso central, o qual pode proporcionar patologias como a microcefalia, hipotonia e hipoplasia do nervo óptico, além de gerar alterações no crescimento intrauterino (CARREAU AM, et al., 2017).

A vitamina $\mathrm{K}$ quando se encontra deficiente na gestante pode contribuir para distúrbios hemodinâmicos, visto que, está relacionada pode causar hemorragias intracranianas e hemorragias durante o período gestacional. Ao mencionar as deficiências do cálcio e vitamina $D$ deve-se considerar que a mineralização fetal é reduzida, levando a um risco de parto prematuro e baixo peso ao nascimento. O mineral zinco está associada ao crescimento fetal, e quando deficiente no organismo materno influência de forma negativa no desenvolvimento fetal, contribui para os risco de malformações congênitas e partos prematuros (CARREAU AM, et al., 2017).

Outro aspecto importante a ser relatado diz respeito ao aumento do risco do parto prematuro, principalmente se a gestação ocorrer durante o primeiro ano após a cirurgia bariátrica. Isso pode ser atribuído ao aumento da perda de peso durante a gravidez, o que pode resultar em desnutrição fetal e parto prematuro. Recomenda-se adiar a gravidez após 24 meses da realização da cirurgia bariátrica, pois as taxas de complicações obstétricas são reduzidas e geram menos riscos aos recém-nascidos (STOPP T, et al., 2018; MALAKAUSKIENE L, et al., 2020). Em decorrência da má absorção, por consequência da cirurgia bariátrica, a composição do leite materno é afetada em diferentes intensidades, o que irá contribuir para a desnutrição fetal, havendo necessidade de usar fórmulas alimentares para o recém-nascido (STOOP T, et al., 2018).

O tamanho de neonatos inferiores a $10^{\circ}$ percentil se demonstra com uma incidência significativa elevada para pacientes que se submeteram a cirurgia bariátrica à medida que se compara com neonatos de gestantes obesas (HAZART J, et al., 2017). Devido à redução nas chances de diabetes mellitus gestacional em mulheres de passaram pelo procedimento de cirúrgico bariátrico, os riscos metabólicos e o menor impacto sobre o desenvolvimento fetais se estende os benefícios para a criança ao longo da vida, o que resulta não apenas no peso adequado ao nascer, mas também no índice de massa corporal durante a infância, além de melhorar os perfis lipídicos e metabólicos na adolescência e fase adulta (MONSON M e JACKSON M, 2016).

\section{CONSIDERAÇÕES FINAIS}

A cirurgia bariátrica ao ser realizada em mulheres em idade reprodutiva está associada a consequências maternas e fetais durante o desenvolvimento do período gestacional. O aconselhamento pré-concepcional e a suplementação das pacientes que apresentam o desejo de engravidar devem ser realizados de acordo com as suas necessidades nutricionais após o procedimento. As contribuições da cirurgia bariátrica podem ser benéficas, visto que, a perda de peso proporciona a redução de comorbidades como a diabetes gestacional e pré-eclâmpsia. Contudo, a ocorrência de complicações decorrentes das mudanças no trato digestivo apresenta malefícios para o desenvolvimento do feto, principalmente, pela redução da absorção de nutrientes no organismo materno que são importantes para a formação do feto. 


\section{REFERÊNCIAS}

1. ABENHAIM HA, et al. Pregnancy outcomes in women with bariatric surgery as compared with morbidly obese women. The Journal of Maternal-Fetal \& Neonatal Medicine, 2018; 29(22): 3596-3601.

2. ADAM S, et al. Pregnancy after bariatric surgery: screening for gestacional diabetes. The BMJ, $2017 ; 356$ : j533.

3. ADAMS T, et al. Resultados maternos e neonatais para gestações antes e depois da cirurgia de redução do estômago. Int J Obes, 2015. 39: 686-694.

4. AKHTER Z, et al. Pregnancy after bariatric surgery and adverse perinatal outcomes: A systematic review and metaanalysis. PLoS Med, 2019; 16(8): e1002866.

5. CARREAU AM, et al. Pregnancy after Bariatric Surgery: Balancing Risks and Benefits. Canadian Journal of Diabetes, 2017; 41(4): 432-438.

6. COELHO A, et al. Gravidez após cirurgia bariátrica. Acta Obstet Ginecol Port, 2020; 14(2): 106-110.

7. COLQUITT J, et al. Surgery for weight loss in adults (Review). Cochrane Database Systematic Reviews, 2014; 8:CD003641.

8. FALCONE V, et al. Pregnancy after bariatric surgery: a narrative literature review and discussion of impact on pregnancy management and outcome. BMC Pregnancy Childbirth, 2018; 18(1): 507.

9. HAZART J, et al. Maternal Nutritional Deficiencies and Small-for-Gestational-Age Neonates at Birth of Women Who Have Undergone Bariatric Surgery. Journal of Pregnancy, 2017; (9): 1-11.

10. HERNANDEZ-PINZON J, et al. Embarazo posterior a cirugía bariátrica: complicaciones maternas y fetales. Rev Colomb Obstet Ginecol, 2008; 59(3): 216-222.

11. MALAKAUSKIENE L, et al. Is it necessary to postpone pregnancy after bariatric surgery: a national cohort study. Journal of Obstetrics and Gynecology, 2020; 40(5): 614-618.

12. MANCINI MC. Dealing with diabetes and pregnancy following bariatric surgery: a double-edged sword?. Archives of Endocrinology and Metabolism, 2016; 60(4): 299-302.

13. MENKE MN, et al. Contraception and Conception After Bariatric Surgery. Obstet Gynecol, 2017; 130(5): $979-987$.

14. MONSON M, JACKSON M. Pregnancy After Bariatric Surgery. Clinical Obstetrics and Gynecology. 2016; 59(1): 15951.

15. NARAYANAN RP, SYED AA. Pregnancy Following Bariatric Surgery-Medical Complications and Management. Obes Surg, 2016; 26(10): 2523-9.

16. ROBSON S, et al. Bariatric Surgery for Women of Reproductive Age. British Journal of Obstetrics and Gynecology, 2015; 123(2): 171-174.

17. RÓŻAŃSKA-WALĘDZIAK A, et al. The Influence of Bariatric Surgery on Pregnancy and Perinatal Outcomes-A CaseControl Study. J Clin Med, 2020; 9(5): 1324.

18. STOOP T, et al. Fertility, Pregnancy and Lactation After Bariatric Surgery - a Consensus Statement from the OEGGG. Geburtshilfe Frauenheilkd, 2018; 78(12): 1207-1211.

19. STUART A, KÜLLEN K. Risk of Abdominal Surgery in Pregnancy Among Women Who Have Undergone Bariatric Surgery. The American College of Obstetricians and Gynecologists, 2017; 129(5): 887-895.

20. UPALA S, SANGUANKEO A. Association between bariatric surgery and pregnancy outcomes. J Matern Fetal Neonatal Med, 2016; 29(17): 2839. 